\title{
Influence of DNA extraction kits on the fungal DNA metabarcoding for freshwater environmental DNA
}

\author{
Shunsuke Matsuoka ${ }^{1}$, Yoriko Sugiyama ${ }^{2}$, Mariko Nagano $^{3}$, Hideyuki Doi ${ }^{1}$ \\ ${ }^{1}$ Graduate School of Information Science, University of Hyogo, Japan \\ ${ }^{2}$ Graduate School of Human and Environmental Studies, Kyoto University, Japan \\ ${ }^{3}$ Department of Bioenvironmental Design, Kyoto University of Advanced Science, Japan
}

Corresponding Author: Shunsuke Matsuoka (code_matuoka@hotmail.com)

\begin{abstract}
Background: Environmental DNA (eDNA) metabarcoding is a rapidly expanding technique for efficient biodiversity monitoring, especially of animals. Recently, the usefulness of aquatic eDNA in monitoring the diversity of both terrestrial and aquatic fungi has been suggested. In eDNA studies, different experimental factors, such as DNA extraction kits or methods, can affect the subsequent analyses and the results of DNA metabarcoding. However, few methodological studies have been carried out on eDNA of fungi, and little is known about how experimental procedures can affect the results of biodiversity analysis. In this study, we focused on the effect of the DNA extraction method on fungal DNA metabarcoding using freshwater samples obtained from rivers and lakes.

Methods: DNA was extracted from freshwater samples using the DNeasy PowerSoil kit, which is mainly used to extract microbial DNA from soil, and the DNeasy Blood \& Tissue kit, which is commonly used for eDNA studies on animals. We then compared PCR inhibition and fungal DNA metabarcoding results [i.e., operational taxonomic unit (OTU) number and composition] of the extracted samples.

Results: No PCR inhibition was detected in any of the samples, and no significant differences in the number of OTUs and OTU compositions were detected between the samples processed using different kits. These results indicate that both DNA extraction kits may provide similar diversity results for the river and lake samples evaluated in this study. Therefore, it may be possible to evaluate the diversity of fungi using a unified experimental method, even with samples obtained for diversity studies on other taxa such as those of animals.
\end{abstract}

\section{Keywords:}

biodiversity, environmental DNA, freshwater, fungi, metabarcoding 


\section{Introduction}

DNA metabarcoding for environmental DNA in water (eDNA metabarcoding) has exploded in recent years as a simple and powerful method for biodiversity assessment and monitoring (Yamamoto et al., 2017; Miya, Gotoh \& Sado, 2020; Doi et al., 2021; Gehri et al., 2021). Studies on eDNA metabarcoding include methodological considerations of experimental procedures and processes, such as sampling and DNA extraction methods (Doi et al., 2019; Coutant et al., 2020; Mathon et al., 2021), as well as those applied to monitoring in various taxonomic groups and ecosystems (Ruppert, Kline \& Rahman, 2019; Mathieu et al., 2020; Tsuji et al., 2020). These experimental conditions have so far been studied mainly for aquatic animals, especially fish, and have contributed to the development of protocols for biodiversity monitoring using eDNA (Laramie et al., 2015; Minamoto et al., 2021). However, there are fewer such methodological studies in fungi, and thus the potential effects of experimental procedures on the results of aquatic eDNA metabarcoding for fungi remain unclear.

Fungi drive ecosystem processes in aquatic and terrestrial habitats through the decomposition of organic matter, parasites, and symbionts of other organisms (Peay, Kennedy \& Talbot, 2016; Grossart et al., 2019). However, the high local diversity and substrate heterogeneity of fungi make it difficult to assess spatial and temporal changes in their diversity, especially on large spatiotemporal scales. Recently, the analysis of fungal DNA in freshwater rivers and lakes has shown the possibility of efficiently assessing fungal diversity in the surrounding ecosystems, including aquatic and terrestrial fungi (Deiner et al., 2016; Khomich et al., 2017; LeBrun et al., 2018; Matsuoka et al., 2019; Matsuoka et al., 2021). Knowledge of the potential effects of experimental procedures on the metabarcoding results is essential for further validation of the usefulness of eDNA analysis in fungal diversity assessment. Among the eDNA experiments, the DNA extraction method is one of the most important factors influencing metabarcoding results (Ushio, 2019). For example, DNA metabarcoding results (species richness and composition detected) can vary depending on the kits or methods used for DNA extraction in experiments with terrestrial substrates (e.g., mycorrhizal fungi; Tedersoo et al., 2010). However, there are no examples of the evaluation of fungal DNA in freshwater.

To date, the major freshwater ecosystems to which eDNA metabarcoding has been applied are rivers, lakes, and ponds. Although they differ in the presence or absence of constant water flow, there are no examples of how these habitat types affect DNA extraction from fungi. For example, it has been reported from experiments with fish DNA collected from environments with low water flow, experimental processing after DNA extraction is inhibited by PCR, probably due to humic acid (Fujii et al., 2019). When inhibition occurs, the number of taxa detected by DNA metabarcoding may be reduced (Fujii et al., 2019; Takasaki et al., 2021). Inhibitors can be removed to some extent by using efficient DNA extraction kits, but the ability to remove them varies among various DNA extraction kits. Therefore, differences in the ability of DNA extraction kits to remove inhibitors may affect the results of DNA metabarcoding.

The purpose of this study was to evaluate the effect of DNA extraction kits on the results of fungal DNA metabarcoding for freshwater samples, with a particular focus on two habitats with 
different characteristics: rivers and lakes/ponds. DNA extraction was carried out on the samples obtained from rivers and lakes using the DNeasy PowerSoil kit and Blood \& Tissue kit (Qiagen, Hilden, Germany). The former kit has a good ability to remove inhibitors such as humic acids and is widely used to extract fungal DNA from soil (Song et al., 2015). The latter is less effective as compared to PowerSoil in its ability to remove inhibitors, but requires fewer steps and is widely used for eDNA studies of aquatic animals (Minamoto et al., 2021). For the eDNA obtained, we first compared the strength of PCR inhibition and then compared the fungal DNA metabarcoding results (i.e., OTU numbers and compositions).

\section{Materials \& Methods}

\section{Sampling and DNA extraction}

One liter of surface water was collected from each of the three rivers [Kamo (Kyoto), Umekoji (Kyoto), and Toga (Hyogo)] and three lakes/ponds [Biwa (Shiga), Takaraga (Kyoto), and Shuhou (Hyogo)] in Japan from April 29 to May 18, 2017 (Fig. 1 and Table S1). Water samples were collected in DNAfree polypropylene bottles. Benzalkonium chloride $(1 \mathrm{~mL}, 10 \% \mathrm{w} / \mathrm{v})$ was added to per liter of water sample immediately after collection to avoid a decrease in eDNA concentration in the samples (Yamanaka et al., 2016). The water samples were kept in a cooler bag with ice packs during transport. The samples were filtered through GF/F glass filters (pore size $0.7 \mu \mathrm{m}$, GE Healthcare, Little Chalfont, UK) immediately after returning to the laboratory. The filters were stored at $-20{ }^{\circ} \mathrm{C}$ until DNA extraction.

For each sample, DNA extraction was performed in three different ways (Fig. 2). First, each filter was cut into three pieces. Further, DNA extraction was performed on each piece using the following methods- (i) DNeasy Blood \& Tissue kit (Qiagen, Hilden, Germany, hereafter referred to as BT), following the same extraction protocol as followed for MiFish analysis (Uchii, Doi \& Minamoto, 2016; Minamoto et al., 2021); (ii) DNeasy Blood \& Tissue kit, after cutting the filter into small pieces using bleached dissecting scissors (referred to as BTC); (iii) DNeasy PowerSoil kit (Qiagen, Hilden, Germany, referred to as PS), after cutting the filter into small pieces using bleached dissecting scissors prior to bead beating. DNA using each kit was extracted according to the manufacturer's instructions.

\section{Molecular experiments and bioinformatics}

Molecular experiments and bioinformatics procedures were the same as those described by Matsuoka et al. (2019) and Matsuoka et al. (2021). Briefly, the first-round PCR (first PCR) amplified the fungal internal transcribed spacer 1 (ITS1) region using the ITS1F-ITS2 primer set (Toju et al., 2012). Eight replicate first PCRs (per sample) were performed, and the duplicated PCR amplicons (per sample) were combined, resulting in a template per sample for the second PCR. A second PCR was conducted to add the index sequence and adapter sequences. Details of the experimental procedure are presented in Appendix 1. Sequencing was performed using MiSeq 250 paired-end 
sequencing at Ryukoku University, Japan. The sequence data were deposited in the Sequence Read Archive of the DNA Data Bank of Japan (Accession: DRA012030).

For all sequences, low-quality sequences with a total length of less than 120 bp were excluded, and forward and reverse sequences were concatenated. Subsequently, potential sequencing errors and chimeric sequences were removed using algorithms in CD-HIT-OTU (Li et al., 2012) and UCHIME v4.2.40 (Edgar et al., 2011), respectively. Operational taxonomic units (OTUs) were generated by clustering the remaining sequences based on $97 \%$ sequence similarity (Osono, 2014). For each of the OTUs, taxonomic assignment was conducted using the query-centric auto-k-nearest-neighbor method (Tanabe \& Toju, 2013) and the lowest common ancestor algorithm (Huson et al., 2007) with the NCBI database. Then, a matrix of OTUs $\times$ samples was constructed (number of reads in the cells, Table S2). For the OTU matrix, cell entries with reads less than $0.0002 \%$ (which corresponds to 2-9 reads, a typically used criterion in eDNA metabarcoding studies, Table S2) of the total number of reads for each sample were replaced with zero because these rare entries could represent contamination. Non-fungal OTUs were excluded from the analysis. No rarefaction was performed on the OTU-sample count curve because almost all the samples were saturated (Fig. S1).

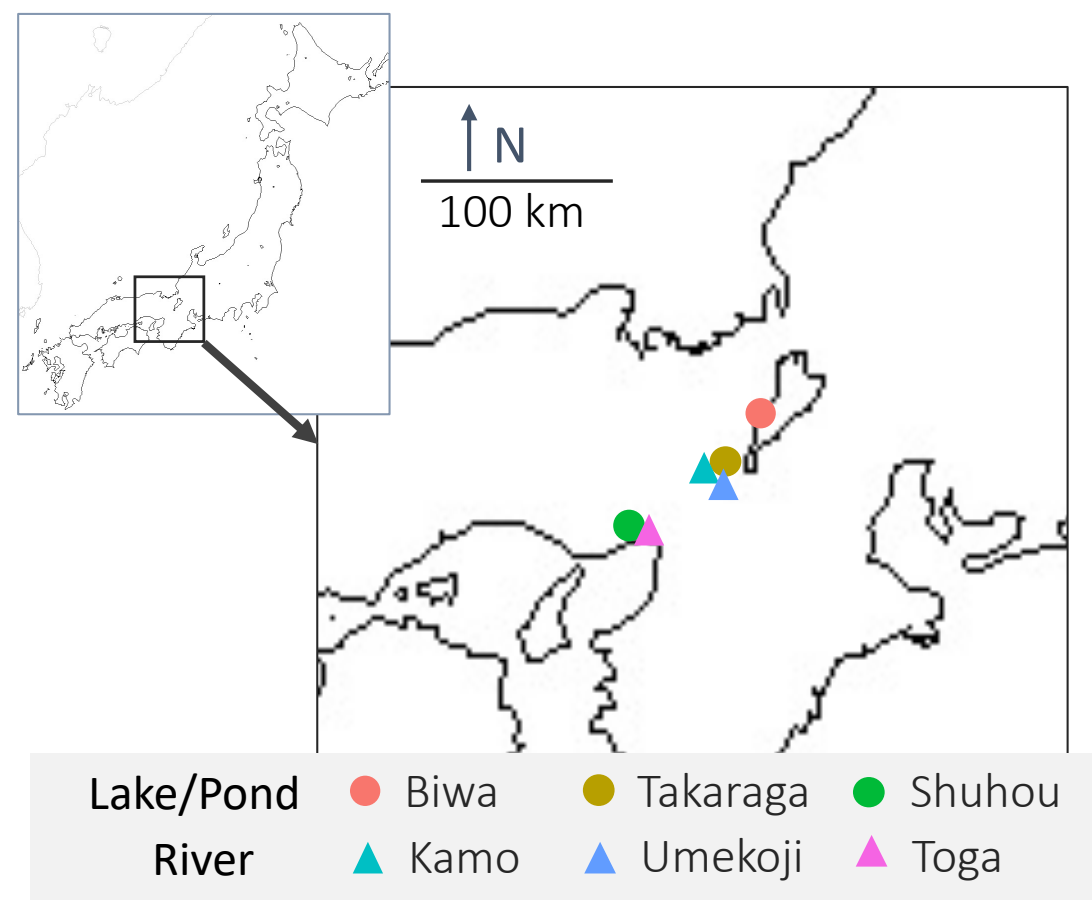

Figure 1 Map of sampling locations and their habitat types 
(1) The filter was cut into three pieces

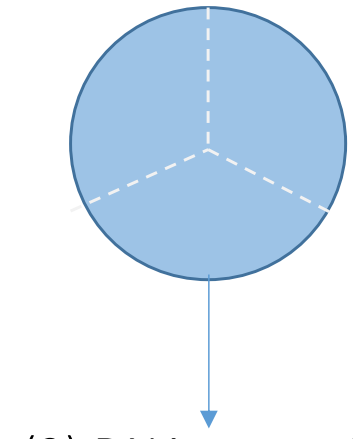

(2) DNA extraction

(i) DNeasy Blood \& Tissue w/o filter chop

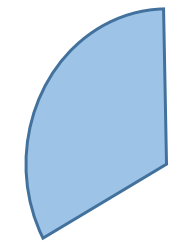

Blood \& Tissue

(ii) DNeasy Blood \& Tissue w/ filter chop

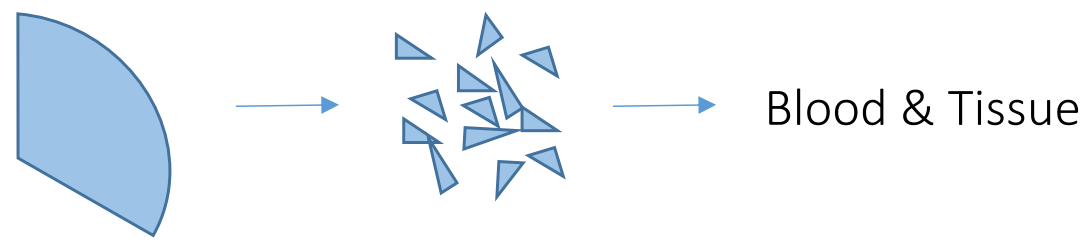

(iii) DNeasy PowerSoil w/ filter chop

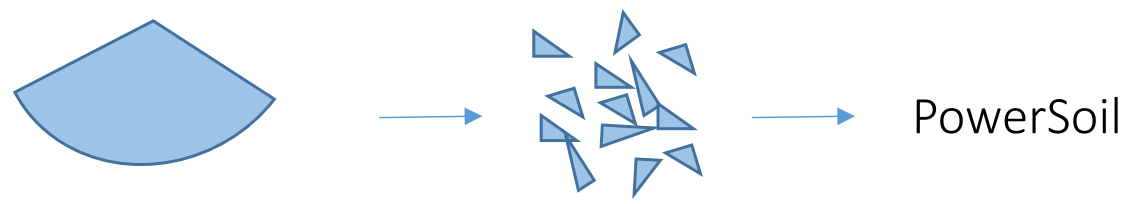

Figure 2 Overview of the DNA extraction procedure. Each filter paper was first divided into three pieces and DNA was extracted using different methods. (i) DNeasy Blood \& Tissue kit without filter chop (ii) DNeasy Blood \& Tissue kit with filter chop (iii) DNeasy PowerSoil kit with filter chop.

\section{PCR inhibition test}

We tested the inhibition of PCR in eDNA samples. Inhibition was evaluated by including $1 \mu \mathrm{L}$ of a plasmid that included an internal positive control (IPC, 207-bp; Nippon Gene Co., Ltd., Tokyo, Japan; $1.5 \times 10^{2}$ copies) in the PCR reaction, instead of DNA-free distilled water, and the following primer-probe set: forward primer, IPC1-5: 5-CCGAGCTTACAAGGCAGGTT-3, reverse primer, IPC1-3: 5-TGGCTCGTACACCAGCATACTAG-3', and TaqMan probe, IPC1-Taq: 5-(FAM)- 
TAGCTTCAAGCATCTGGCTGTCGGC-(TAMRA)-3, which contained $900 \mathrm{nM}$ of each primer and $125 \mathrm{nM}$ of TaqMan probe in a 1× PCR master mix (KOD FX Neo, TOYOBO, Osaka, Japan) and 2 $\mu \mathrm{L}$ of the DNA solution. The total volume of each reaction mixture was $10 \mu \mathrm{L}$. Real-time PCR was performed using quantitative real-time PCR (PikoReal real-time PCR, Thermo Fisher Scientific). PCR (three replicates) was performed as follows: $2 \mathrm{~min}$ at $50^{\circ} \mathrm{C}, 10 \mathrm{~min}$ at $95^{\circ} \mathrm{C}$, and $55 \mathrm{cycles}$ of $15 \mathrm{~s}$ at $95^{\circ} \mathrm{C}$, and $60 \mathrm{~s}$ at $60^{\circ} \mathrm{C}$. Non-template control (NTC) was performed in three replicates per PCR. The PCR results were analyzed using PikoReal software v. 2.2.248.601 (Thermo Fisher Scientific). The presence of PCR inhibitors was evaluated using $\Delta \mathrm{Ct}\left(\mathrm{Ct}_{\text {positive control }}-\mathrm{Ct}_{\text {sample }}\right) . \Delta \mathrm{Ct}$ of $\geq 3$ cycles and is usually considered to be evidence of inhibition (Hartman, Coyne \& Norwood, 2005).

\section{Data analysis}

All analyses were performed using R v.3.4.3 (R Core Team, 2017). Differences in the number of OTUs between the DNA extraction kit and sampling site were evaluated. First, the effect of differences in the DNA extraction procedures was investigated using the generalized linear mixed model (GLMM) using the glmer function in the Ime4 package. The error structure was a Poisson distribution, and sampling sites were specified as random terms. Next, a post-hoc test using Tukey's HSD method was performed to determine significantly different extraction procedures. The effect of sampling sites was then examined using the generalized linear model (GLM) using the glm function. The error structure is a Poisson distribution. Differences in the OTU composition by DNA extraction procedures and sampling sites were evaluated by permutational multivariate analysis of variance (PERMANOVA), using the adonis2 function in the vegan package ver. 2.5-6 with 9999 permutations. OTU compositions were evaluated using three dissimilarity indices: Bray-Curtis, Jaccard, and RaupCrick indices. For the Bray-Curtis index, the OTU matrix containing the relative number of reads for each OTU per sample was converted into a dissimilarity matrix. For the Jaccard and Raup-Crick indices, the OTU matrix containing the presence/absence of OTUs per sample was used. The RaupCrick index is known to be less affected by differences in the number of OTUs between samples than the Bray-Curtis and Jaccard indices (Chase et al, 2011). Variations in OTU composition were visualized using non-metric multidimensional scaling (NMDS) using the phyloseq package, and factors associated with the NMDS ordinations (DNA extraction procedures (PS, BT, or BTC), habitats (river or pond/lake), and latitude and longitude) were evaluated using the envfit function in the vegan package.

Indicator taxa analysis (De Cáceres \& Legendre, 2009) was performed to determine which OTUs had significantly different frequencies between DNA extraction kits. The analysis was performed using the signassoc function in the indicspecies package ver. 1.7.8 (De Cáceres \& Legendre, 2009) on the two OTU matrices: the relative number of reads for each OTU per sample and the presence/absence of data. We used mode $=1$ (group-based) and calculated the P-values with 999 permutations after Sidak's correction of multiple testing. 


\section{Results and Discussion}

A total of 1,362 OTUs were detected in 18 samples ( 6 sites $\times 3$ DNA extraction conditions) (15-372 OTUs per sample, mean 134 OTUs). Both DNA extraction and the study site significantly affected the number of OTUs (DNA extraction: GLMM, P $<0.0001$; site: GLM, P $<0.0001$ ). There was no significant difference in the number of OTUs detected between the PowerSoil kit (PS) and the Blood \& Tissue kit (BT) (Fig. 3, GLMM, Tukey's HSD, P = 0.295), but when the Blood \& Tissue kit was subjected to filter chopping (BTC), the number of OTUs detected was significantly lower than that of PS or BT (Fig. 3, GLMM, Tukey's HSD, P $<0.05$ ).

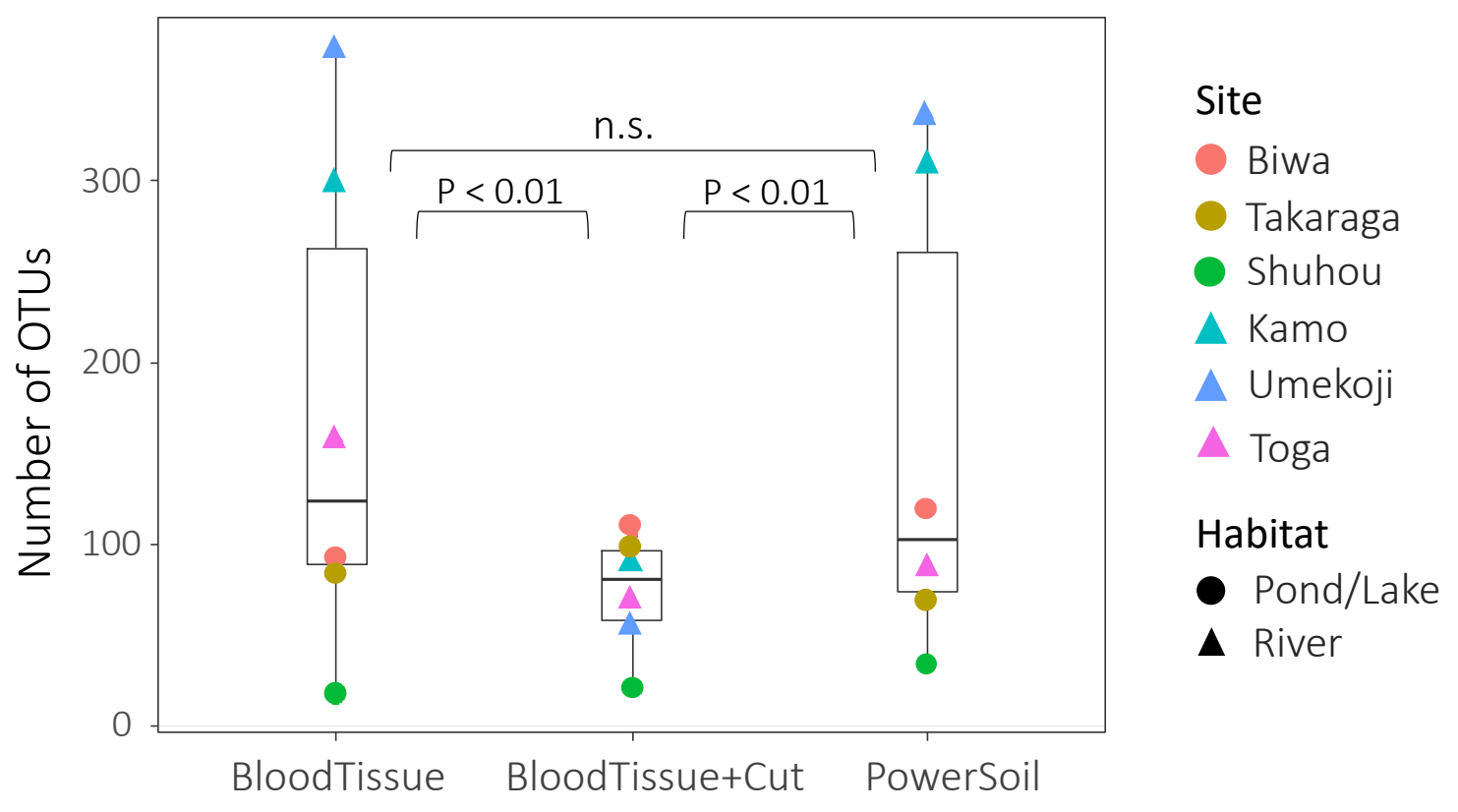

Figure 3 Box plots showing the number of OTUs for each DNA extraction kit. Points indicate the number of OTUs per study site. Although no significant difference was detected between PowerSoil and Blood Tissue kits (GLMM, Tukey's HSD, P > 0.05), the number of operational taxonomic units (OTUs) was significantly lower in the Blood Tissue + Cut samples than in the other two treatments (GLMM, Tukey's HSD, P < 0.01).

For OTU composition, no effect of DNA extraction condition was detected for Bray-Curtis, Jaccard, or Raup-Crick indices (PERMANOVA, P > 0.900), and OTU compositions were significantly similar for the same study sites (PERMANOVA, $\mathrm{P}<0.0001$ ). The results of the NMDS ordination showed a similar trend (Fig. 4). No significant relationship with the DNA extraction method was detected (envfit, $\mathrm{P}>0.05$ ), and only habitat type was significantly related to the ordination (envfit, $P<0.001$, Fig. 4). The variation within the same study site (i.e., variations among DNA extraction methods) was smaller for the Raup-Crick index than for the Bray-Curtis or Jaccard indices (Fig. 4), suggesting that the variation in OTU compositions among the extraction methods at the same study 
site was mainly due to differences in the number of detected OTUs rather than OTU turnover because the Raup-Crick index was less affected by alpha diversity gradients among samples than the Bray-Curtis or Jaccard indices (Chase et al., 2011). Furthermore, no individual OTUs were found to be significantly more abundant in a particular DNA extraction kit (indicator taxa analysis, $\mathrm{P}>$ 0.05, Table S3). These results indicate that the effect of the DNA extraction kit on the number of detected OTUs and OTU composition is limited, and that both DNA extraction kits may provide similar results that reflect differences in sites and habitats, especially in the analysis of OTU richness and composition.

(a) Bray-Curtis

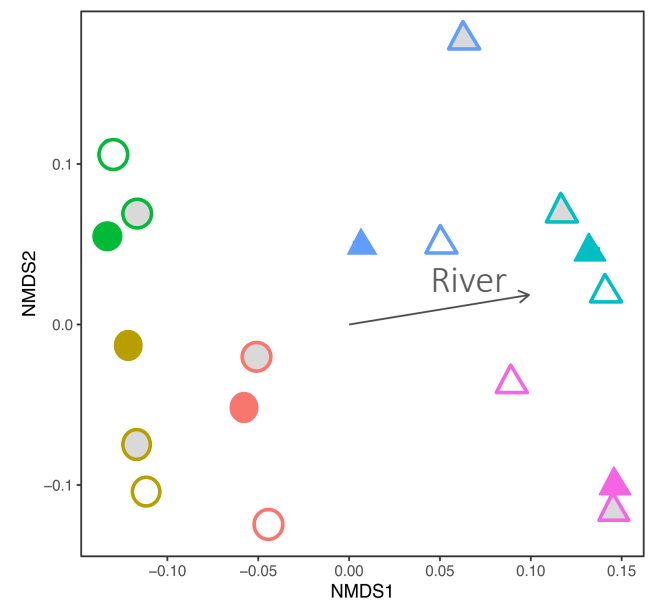

(c) Raup-Crick

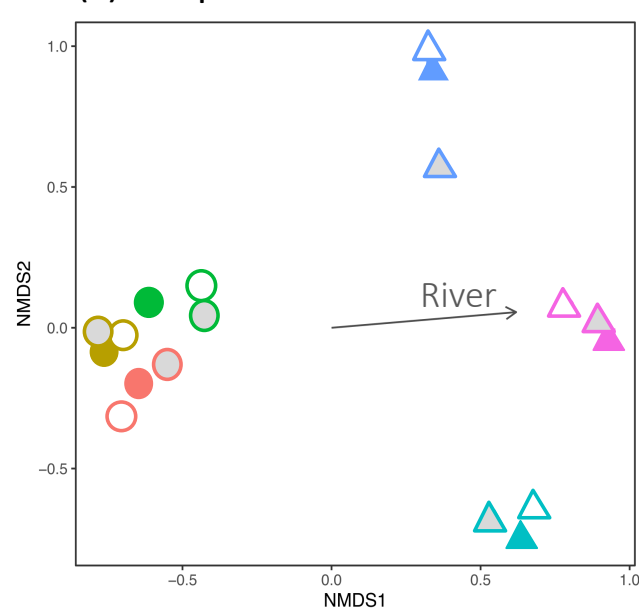

(b) Jaccard

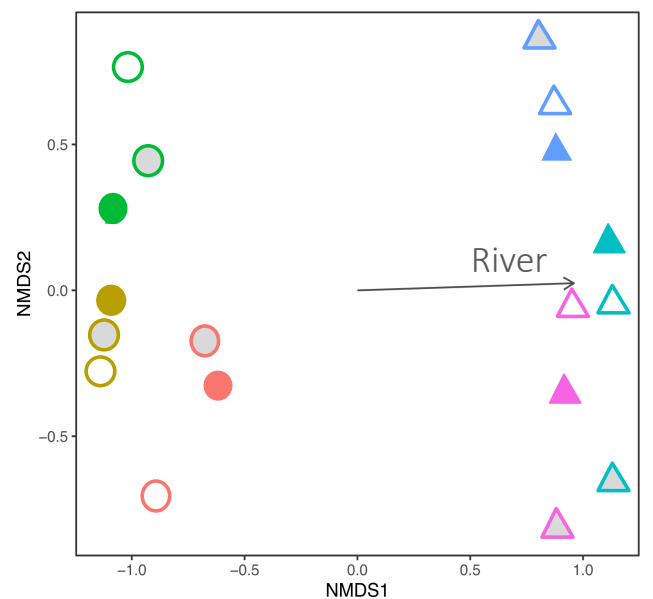

Site

- Biwa O BloodTissue

Takaraga

BloodTissue+Cut

Shuhou

- PowerSoil

A Kamo

$\Delta$ Umekoji

Habitat

$\Delta$ Toga

- Pond/Lake

$\Delta$ River

Figure 4 Dissimilarity in the fungal DNA assemblages among samples, as revealed via non metric multidimensional scaling (NMDS) ordination (a) Bray-Curtis index, stress $=0.1402$, (b) Jaccard index, stress $=0.1048$, (c) Raup-Crick index, stress $=0.1245$. For all dissimilarity indices, only habitat type was significantly related to the ordination (envfit, $\mathrm{P}<0.05$ ), and DNA extraction kit and latitude/longitude were not significantly related (envfit, $\mathrm{P}>0.05$ ). 
Differences in DNA extraction methods and kits have been shown to affect the fungal OTU composition in terrestrial substrates (Tedersoo et al., 2010). On the other hand, for fungal DNA in freshwater environments, comparable OTU composition data may be obtained between the DNA extraction kits used in this study. The PowerSoil kit has a superior ability to physically disrupt fungal cell walls and remove PCR inhibitors, although the experimental procedure is more complicated than the Blood \& Tissue kit. Therefore, fungal DNA in terrestrial substrates, especially in soil, is often extracted using the PowerSoil kit. Blood \& Tissue kits have recently been used for eDNA analysis, especially in animals (Minamoto et al., 2021). The results of the present study indicate that fungal eDNA in freshwater can be extracted using the Blood \& Tissue kit, and that the same extracted sample can be used for diversity analysis of multiple taxonomic groups such as animals and fungi. However, when DNA extraction is performed with the Blood \& Tissue kit, the number of OTUs detected may decrease when the filter is chopped up. The reason for the decrease in the number of OTUs detected by the paper chopping process is not clear in this study, but it is possible that the finer filter paper tends to clog the column, thereby reducing the efficiency of DNA extraction. It is better to follow the existing eDNA protocol (Uchii, Doi \& Minamoto, 2016; Minamoto et al., 2021) when using the Blood \& Tissue kit.

PCR inhibitor tests using real-time PCR showed that the range of the average $\triangle C T$ per site is from -0.28 to 0.38 (all values are shown in Table S4), indicating that there is no inhibition in the samples ( $\Delta \mathrm{Ct} \geq 3$ is usually considered to be evidence of inhibition, Hartman, Coyne \& Norwood, 2005). This result indicates that PCR inhibition may not be affected by the DNA extraction kit in the present water samples. However, in eDNA studies on freshwater fish in backwater lakes, PCR inhibition, which may be due to high concentrations of organic matter, has been detected using the Blood \& Tissue kit (Fujii et al., 2019). In such cases, the PowerSoil kit may be more suitable because of its superior ability to remove inhibitors. In the future, it would be better to evaluate whether the extraction kit causes any difference in PCR inhibition in freshwater with high organic matter concentrations, such as backwater lakes.

It should be noted that the number of samples and sites used in this study was limited. Therefore, the generality of the results must be verified further. For example, there was no significant difference in the composition of OTUs detected by the extraction kit or inhibition of PCR at the study site in this study, but it is necessary to verify the generality and applicability of the results by targeting water samples under various conditions in the future.

\section{Acknowledgements}

MiSeq sequencing was conducted in the Department of Environmental Solution Technology, Faculty of Science and Technology, Ryukoku University. We thank Hiroki Yamanaka and Hirotoshi Sato for supporting MiSeq sequencing. This study received partial financial support from the Japan Society for the Promotion of Science (JSPS) to SM (20J01732) and the Environmental Research and Technology Development Fund (JPMEERF20164002). 


\section{References}

Chase JM, Kraft NJB, Smith KG, Vellend M, Inouye BD. 2011. Using null models to disentangle variation in community dissimilarity from variation in $\alpha$-diversity. Ecosphere 2:1-11 DOI: 10.1890/ES10-00117.1.

Coutant O, Cantera I, Cilleros K, Dejean T, Valentini A, Murienne J, Brosse S. 2021. Detecting fish assemblages with environmental DNA: Does protocol matter? Testing eDNA metabarcoding method robustness. Environmental DNA 3:619-630 DOI: 10.1002/edn3.158.

De Cáceres MD, Legendre P. 2009. Associations between species and groups of sites: Indices and statistical inference. Ecology 90:3566-3574 DOI: 10.1890/08-1823.1.

Deiner K, Fronhofer EA, Mächler E, Walser JC, Altermatt F. 2016. Environmental DNA reveals that rivers are conveyer belts of biodiversity information. Nature Communications 7:1-9 DOI: 10.1038/ncomms12544.

Doi H, Fukaya K, Oka SI, Sato K, Kondoh M, Miya M. 2019. Evaluation of detection probabilities at the water-filtering and initial PCR steps in environmental DNA metabarcoding using a multispecies site occupancy model. Scientific Reports 9:1-8 DOI: 10.1038/s41598-01940233-1.

Doi H, Inui R, Matsuoka S, Akamatsu Y, Goto M, Kono T. 2021. Estimation of biodiversity metrics by environmental DNA metabarcoding compared with visual and capture surveys of river fish communities. Freshwater Biology 66:1257-1266 DOI: 10.1111/fwb.13714.

Edgar RC, Haas BJ, Clemente JC, Quince C, Knight R. 2011. UCHIME improves sensitivity and speed of chimera detection. Bioinformatics 27:2194-2200 DOI: 10.1093/bioinformatics/btr381.

Fujii K, Doi H, Matsuoka S, Nagano M, Sato H, Yamanaka H. 2019. Environmental DNA metabarcoding for fish community analysis in backwater lakes: A comparison of capture methods. PLOS ONE 14:e0210357 DOI: 10.1371/journal.pone.0210357.

Gehri RR, Larson WA, Gruentha IK, Sard NM, Shi Y. 2021. eDNA metabarcoding outperforms traditional fisheries sampling and reveals fine-scale heterogeneity in a temperate freshwater lake. Environmental DNA 3:912-929 DOI: 10.1002/edn3.197.

Grossart HP, Van den Wyngaert S, Kagami M, Wurzbacher C, Cunliffe M, Rojas-Jimenez K. 2019. Fungi in aquatic ecosystems. Nature Reviews Microbiology 17:339-354 DOI: 10.1038/s41579-019-0175-8.

Hartman LJ, Coyne SR, Norwood DA. 2005. Development of a novel internal positive control for Taqman based assays. Molecular and Cellular Probes 19:51-59 DOI: 10.1016/j.mcp.2004.07.006.

Huson DH, Auch AF, Qi J, Schuster SC. 2007. MEGAN analysis of metagenomic data. Genome Research 17:377-386 DOI: 10.1101/gr.5969107.

Khomich M, Davey ML, Kauserud H, Rasconi S, Andersen T. 2017. Fungal communities in Scandinavian lakes along a longitudinal gradient. Fungal Ecology 27:36-46 DOI: 10.1016/j.funeco.2017.01.008.

Laramie MB, Pilliod DS, Goldberg CS, Strickler KM. 2015. Environmental DNA sampling protocol_Filtering water to capture DNA from aquatic organisms. Reston: U.S. Geological Survey. DOI: $\underline{10.3133 / \mathrm{tm} 2 \mathrm{~A} 13 .}$. 
LeBrun ES, Taylor DL, King RS, Back JA, Kang S. 2018. Rivers may constitute an overlooked avenue of dispersal for terrestrial fungi. Fungal Ecology 32:72-79 DOI: 10.1016/j.funeco.2017.12.003.

Li W, Fu L, Niu B, Wu S, Wooley J. 2012. Ultrafast clustering algorithms for metagenomic sequence analysis. Briefings in Bioinformatics 13:656-668 DOI: 10.1093/bib/bbs035.

Mathieu C, Hermans SM, Lear G, Buckley TR, Lee KC, Buckley HL. 2020. A systematicreview of sources of variability and uncertainty in eDNA data for environmental monitoring. Frontiers in Ecology and Evolution 8:135. DOI: 10.3389/fevo.2020.00135.

Mathon L, Valentini A, Guérin PE, Normandeau E, Noel C, Lionnet C, Boulanger E, Thuiller W, Bernatchez L, Mouillot D, Dejean T, Manel S. 2021. Benchmarking bioinformatic tools for fast and accurate eDNA metabarcoding species identification. Molecular Ecology Resources 21:2565-2579. DOI: 10.1111/1755-0998.13430.

Matsuoka S, Sugiyama Y, Sato H, Katano I, Harada K, Doi H. 2019. Spatial structure of fungal DNA assemblages revealed with eDNA metabarcoding in a forest river network in western Japan. Metabarcoding and Metagenomics 3:37-47 DOI: 10.3897/mbmg.3.36335.

Matsuoka S, Sugiyama Y, Shimono Y, Ushio M, Doi H. 2021. Evaluation of seasonal dynamics of fungal DNA assemblages in a flow-regulated stream in a restored forest using eDNA metabarcoding. Environmental Microbiology 23:4797-4806 DOI: $\underline{10.1111 / 1462-}$ 2920.15669 .

Minamoto T, Miya M, Sado T, Seino S, Doi H, Kondoh M, Nakamura K, Takahara T, Yamamoto S, Yamanaka H, Araki H, Iwasaki W, Kasai A, Masuda R, Uchii K. 2021. An illustrated manual for environmental DNA research: Water sampling guidelines and experimental protocols. Environmental DNA 3:8-13 DOI: 10.1002/edn3.121.

Miya M, Gotoh RO, Sado T. 2020. MiFish metabarcoding: A high-throughput approach for simultaneous detection of multiple fish species from environmental DNA and other samples. Fisheries Science 86:939-970 DOI: 10.1007/s12562-020-01461-x.

Osono T. 2014. Metagenomic approach yields insights into fungal diversity and functioning. In: SotaT, KagataH, AndoY, UtsumiS, OsonoT, eds. Species diversity and community structure. Tokyo: Springer, 1-23 DOI: 10.1007/978-4-431-54261-2_1.

Peay KG, Kennedy PG, Talbot JM. 2016. Dimensions of biodiversity in the Earth mycobiome. Nature Reviews Microbiology 14:434-447 DOI: 10.1038/nrmicro.2016.59.

R Core Team. 2017. R: A language and environment for statistical computing. Available at https://www.R-project.org/.

Ruppert KM, Kline RJ, Rahman MS. 2019. Past, present, and future perspectives of environmental DNA (eDNA) metabarcoding: A systematic review in methods, monitoring, and applications of global eDNA. Global Ecology and Conservation 17:e000547 DOI: 10.1016/j.gecco.2019.e00547.

Song Z, Schlatter D, Kennedy P, Kinkel LL, Kistler HC, Nguyen N, Bates ST. 2015. Effort versus reward: Preparing samples for fungal community characterization in high-throughput sequencing surveys of soils. PLOS ONE 10:e0127234 DOI: 10.1371/journal.pone.0127234. 
Takasaki K, Aihara H, Imanaka T, Matsudaira T, Tsukahara K, Usui A, Osaki S, Doi H. 2021. Water pre-filtration methods to improve environmental DNA detection by real-time PCR and metabarcoding. PLOS ONE 16:e0250162 DOI: 10.1371/journal.pone.0250162.

Tanabe AS, Toju H. 2013. Two new computational methods for universal DNA barcoding: A benchmark using barcode sequences of bacteria, archaea, animals, fungi, and land plants. PLOS ONE 8:e76910 DOI: 10.1371/journal.pone.0076910.

Tedersoo L, Nilsson RH, Abarenkov K, Jairus T, Sadam A, Saar I, Bahram M, Bechem E, Chuyong G, Kõljalg U. 2010. 454 pyrosequencing and Sanger sequencing of tropical mycorrhizal fungi provide similar results but reveal substantial methodological biases. New Phytologist 188:291-301 DOI: 10.1111/j.1469-8137.2010.03373.x.

Toju H, Tanabe AS, Yamamoto S, Sato H. 2012. High-coverage ITS primers for the DNA-based identification of ascomycetes and basidiomycetes in environmental samples. PLOS ONE 7:e40863 DOI: 10.1371/journal.pone.0040863.

Tsuji S, Takahara T, Doi H, Shibata N, Yamanaka H. 2019. The detection of aquatic macroorganisms using environmental DNA analysis-A review of methods for collection, extraction, and detection. Environmental DNA 1:99-108 DOI: 10.1002/edn3.21.

Uchii K, Doi H, Minamoto T. 2016. A novel environmental DNA approach to quantify the cryptic invasion ofnon-native genotypes. Molecular Ecology Resources 16:415-422 DOI: 10.1111/1755-0998.12460.

Ushio M. 2019. Use of a filter cartridge combined with intra-cartridge bead-beating improves detection of microbial DNA from water samples. Methods in Ecology and Evolution 10:11421156 DOI: $10.1111 / 2041-210 X .13204$.

Yamamoto S, Masuda R, Sato Y, Sado T, Araki H, Kondoh M, Minamoto T, Miya M. 2017. Environmental DNA metabarcoding reveals local fish communities in a species-rich coastal sea. Scientific Reports 7:1-2 DOI: 10.1038/srep40368.

Yamanaka H, Minamoto T, Matsuura J, Sakurai S, Tsuji S, Motozawa H, Hongo M, Sogo Y, Kakimi N, Teramura I, Sugita M, Baba M, Kondo A. 2017. A simple method for preserving environmental DNA in water samples at ambient temperature by addition of cationic surfactant. Limnology 18:233-241 DOI: 10.1007/s10201-016-0508-5.

\section{Supporting Information}

Table S1 Details of sampling sites and sampling.

Table S2 List of all OTUs, the number of their sequence reads and consensus sequence, and taxonomic assignments by Claident on GenBank.

Table S3 Results of indicator taxa analysis for all OTUs. Best means preferred methods. The p-values were calculated with 999 permutations after Sidak's correction of the multiple testing.

Table S4 List of delta CT values for all samples.

Figure S1 Relationship between the number of sequence reads and OTU numbers, i.e., rarefaction curves for the samples. 


\section{Appendix 1. The detailed procedure of molecular analyses}

For MiSeq sequencing, the fungal internal transcribed spacer 1 (ITS 1) region of rDNA was amplified. The first-round PCR (first PCR) amplified the ITS1 region using the ITS1-F-KYO2 and ITS2-KYO2 primer set, which is capable of amplifying the ITS1 region of most fungal groups. An Illumina sequencing primer and six random bases $(\mathrm{N})$ were combined to produce each primer. Thus, the forward primer sequence was: 5'- ACA CTC TTT CCC TAC ACG ACG CTC TTC CGA TCT NNNNNN TAG AGG AAG TAA AAG TCG TAA -3' and the reverse primer sequence was: 5'- GTG ACT GGA GTT CAG ACG TGT GCT CTT CCG ATC T NNNNNN TTY RCT RCG TTC TTC ATC- 3'. The italic and normal letters represent MiSeq sequencing primers and fungi-specific primers, respectively. The six random bases $(\mathrm{N})$ were used to enhance cluster separation on the flowcells during initial base call calibrations on MiSeq.

The $1^{\text {st }}$ PCR was performed in a $12 \mu$ volume with the buffer system of KODFX NEO (TOYOBO, Osaka, Japan), which contained $2.0 \mu \mathrm{l}$ of template DNA, $0.2 \mu \mathrm{l}$ of KOD FX NEO, $6.0 \mu \mathrm{l}$ of $2 \times$ buffer, $2.4 \mu \mathrm{l}$ of $\mathrm{dNTP}$, and $0.7 \mu \mathrm{l}$ each of the two primers $(5 \mu \mathrm{M})$. The PCR conditions were as follows; an initial incubation for $2 \mathrm{~min}$ at $94^{\circ} \mathrm{C}$ followed by 5 cycles of $10 \mathrm{~s}$ at $98^{\circ} \mathrm{C}, 30 \mathrm{~s}$ at $68^{\circ} \mathrm{C}$ for annealing and $30 \mathrm{~s}$ at $68^{\circ} \mathrm{C}, 5$ cycles of $10 \mathrm{~s}$ at $98^{\circ} \mathrm{C}, 30 \mathrm{~s}$ at $65^{\circ} \mathrm{C}$ and $30 \mathrm{~s}$ at $68^{\circ} \mathrm{C} ; 5$ cycles of $10 \mathrm{~s}$ at $98^{\circ} \mathrm{C}, 30 \mathrm{~s}$ at $62^{\circ} \mathrm{C}$ and $30 \mathrm{~s}$ at $68^{\circ} \mathrm{C} ; 25$ cycles of $10 \mathrm{~s}$ at $98^{\circ} \mathrm{C}, 30 \mathrm{~s}$ at $59^{\circ} \mathrm{C}$ and $30 \mathrm{~s}$ at $68^{\circ} \mathrm{C}$, and a final extension of $5 \mathrm{~min}$ at $68^{\circ} \mathrm{C}$. Eight replicate first-PCRs (per sample) were performed to mitigate the reaction-level PCR bias. Then, the duplicated first PCR amplicons (per sample) were combined, resulting in a template per sample for the second PCR. The PCR templates were purified using Agencourt AMPure XP (PCR product: AMPure XP beads = 1:0.8; Beckman Coulter, Brea, California, USA) before the second PCR.

The second PCR amplified the first PCR amplicons using the primers (forward) 5'-AAT GAT ACG GCG ACC ACC GAG ATC TAC AC XXXXXXXX TCG TCG GCA GCG TCA GAT GTG TAT AAG AGA CAG-3' and (reverse) 5'-CAA GCA GAA GAC GGC ATA CGA GAT XXXXXXXX GTC TCG TGG GCT CGG AGA TGT GTA TAA GAG ACA G- 3'. The italic and normal letters represent the MiSeqP5/P7 adapter and sequencing primers, respectively. The $8 \mathrm{X}$ bases represent dual-index sequences inserted to identify different samples. The second PCR was carried out with $12 \mu$ reaction volume containing $1.0 \mu \mathrm{l}$ of template, $6 \mu \mathrm{l}$ of $2 \times$ KAPA HiFi HotStart ReadyMix (KAPA Biosystems, Wilmington, Washington, USA), $1.4 \mu \mathrm{l}$ of each primer $(2.5 \mu \mathrm{M})$, and $2.2 \mu \mathrm{l}$ of sterilized distilled water. The PCR conditions were as follows; an initial incubation for $3 \mathrm{~min}$ at $95^{\circ} \mathrm{C}$ followed by 12 cycles of $20 \mathrm{~s}$ at $98^{\circ} \mathrm{C}, 15 \mathrm{~s}$ at $72^{\circ} \mathrm{C}$ for annealing and extension, and a final extension of $5 \mathrm{~min}$ at $72^{\circ} \mathrm{C}$.

The indexed second PCR amplicons were pooled to make a library to be sequenced on MiSeq. The volume of each sample added to the library was adjusted to normalize the concentrations of each second PCR product. The pooled library was purified using Agencourt AMPure XP. A target-sized DNA of the purified library (approximately 380-510 base pairs [bp]) was then excised using E-Gel SizeSelect (ThermoFisher Scientific, Waltham, MA, USA). The doublestranded DNA concentration of the library was then adjusted to $4 \mathrm{nmol} / \mathrm{L}$ using Milli-Q water, and the DNA sample was applied to the Illumina MiSeq platform at Ryukoku University, Japan. 